\title{
Root Resorption in Orthodontic Treatment: Scoping Review
}

\author{
Dr Neeta Aryal," Dr Mao Jing² \\ 'Resident, ${ }^{2}$ Professor, Dept of Orthodontics, Tongji Medical College \\ Huazhong University of Science \& Technology, Wuhan, China \\ Correspondence: Dr Neeta Aryal; Email: neeta_aryal@hotmail.com
}

\section{ABSTRACT}

Introduction: Root resorption is the loss of apical root tissue leading to the shortness of root which is often evident in orthodontic tooth movement. Proper management during orthodontic treatment however can minimize this undesirable outcome. The present article attempts to review the etiology of root resorption, methods of diagnosis, and strategies for prevention.

Materials \& Method: A scoping review was done with the purpose to carry out the narrative integration of the relevant evidences on root resorption and orthodontic treatment from the published literatures. The resulting papers were studied and reviewed thoroughly for the key explanation of root resorption in orthodontic patients. A total of 41 published research articles were reviewed.

Discussion: According to the literatures root resorption is common iatrogenic outcome in orthodontic treatment. Biological, mechanical, and combined biological and mechanical factors result in external root resorption. Though most clinicians diagnose root resorption by conventional radiography, researches have clearly shown that CBCT is the promising tool. The clinicians need to counsel orthodontic patients and their parents that the root resorption might be a potential consequence of the treatment lasting for long time. In case of severity; it is essential to reassess the patient and minimize the underlying cause. It is necessary to understand the role of orthodontist in preventing root resorption.

Key words: orthodontic treatment, root resorption

\section{INTRODUCTION}

Physiological root resorption is a normal phenomenon for exfoliation of deciduous tooth. The phenomenon of the root resorption can be compared with scar resulting after undergoing a surgery. However, the root resorption in permanent teeth is an unfortunate outcome and is considered as the pathologic phenomenon.' Root resorption can be defined as the loss of apical root tissue leading to shortness of the root which is often evident in every orthodontic tooth movement. As a natural process in course of tooth movement; forces are distributed through the tooth to the periodontal ligament resulting in generation and influx of osteoclasts leading to the bone resorption. Due to this movement, the osteoblasts differentiate and deposition of bone occurs resulting in imbalance between resorption and deposition; which contribute to the root resorption. ${ }^{2}$ According to the literatures, patients undergoing orthodontic treatment are more likely to suffer root resorption. Hence the evidences generated from research help to insight in understanding the adverse effects of root resorption and aid in decreasing the occurrences. ${ }^{3-5}$

\section{MATERIALS AND METHOD}

The scoping review includes published studies till March 2017. Various journals, academic publications were reviewed for better understanding of the topic using the key words 'root resorption' and 'orthodontics treatment' in different electronic databases. A total of 1059 published articles were retrieved on key words; out of which 41 articles with full text were selected for review. The resulting papers were studied and reviewed thoroughly for the key explanation of root resorption in orthodontic patients.

\section{DISCUSSION}

\section{Internal root resorption}

Internal root resorption is the consequence of trauma or caries with the bacterial extension to the pulp. Two distinct morphological patterns of internal resorption have been described as: radial pulp enlargement resorption and metaplastic (replacement) pulp resorption. Both share common etiologic factors and probably originate from similar pathogenesis with different morphological characteristics. $^{6-12}$ 


\section{External apical root resorption}

External apical root resorption (EARR) is self-limiting in nature and is not detected clinically or radiolographically. ${ }^{13,14}$ It results from physiological responses like pressure due to chewing and muscle forces on the teeth. ${ }^{15,16}$ It needs to be well demarcated from transient apical breakdown (TAB) which results from trauma or due to orthodontic treatment. ${ }^{17}$

Orthodontic treatment can result in EARR and is considered as a common phenomenon following the treatment beginning in the early leveling stages. There is variation in reports about its prevalence. The mean root shortening as a consequence of treatment ranges between 0.5-3 mm. ${ }^{18}$ EARR is a complication of orthodontic treatment resulting in lifelong loss of tooth structure from the root apex. Proper management during orthodontic treatment however can avoid this undesirable outcome. The available literature illustrates that the etiology of EARR is due to multiple factors consisting of biological features, genetic inheritance and effect of orthodontic force. ${ }^{19}$ It primarily occurs in maxillary anterior teeth with an average of $1.4 \mathrm{~mm}$ where worst occurrence is manifested in maxillary lateral incisors. ${ }^{20}$ The orthodontic patients who exhibit detectable root resorption during early six months of active treatment are more likely to exhibit root resorption in the following six month period. ${ }^{21}$

About $80 \%$ patients undergoing orthodontic treatment might develop some degree of EARR. ${ }^{22}$ Hence it is essential to understand the role of orthodontic treatment in resulting EARR as it is a frequent iatrogenic problem. ${ }^{23}$ Over one third of the patients affected with EARR suffer resorption greater than $3 \mathrm{~mm}$, however the severe resorption (greater than $5 \mathrm{~mm}$ ) affects only $2-5 \% .^{24}$

Thus it is essential to assess the risk factors of EARR that can induce the resorption in orthodontic patients to avoid further complications. Reviewing of the related literature contributes to disseminate quality information that can help to minimize the root resorption observed in EARR. ${ }^{25}$

\section{Etiology of apical root resorption}

The etiology of EARR resulting from orthodontic treatment is multi-factorial constituting the wide range of environmental and host factors. They can be categorized as: biological, mechanical, and combined biological and mechanical factors. As per the risk factors for EARR are concerned, individual susceptibility is one of the important factors which can occur in both deciduous and permanent dentitions. ${ }^{26}$

\section{Biological factors}

Genetics: Genetic factor is considered as one of the important determinant for EARR. ${ }^{26}$ As per the research findings, genetic factors account around $50 \%$ variations in tooth resorption. ${ }^{27}$

Systematic factors: Researchers have outlined the role of systemic factors resulting in root resorption. The allergic patients are likelihood of having higher risk. Likewise, lack of estrogens might induce quick orthodontic movement and calcitonin inhibits odontoclastic activities, ${ }^{27}$ and asthma precipitates greater risk to apical root resorption. ${ }^{28}$

Chronological age: With the advancement of age; changes in periodontal membrane occur causing the tissue narrower, less vascularised and aplastic. Meanwhile, alveolar bone becomes denser, less vascularised and aplastic; while cementum becomes wider with age increasing susceptibility to bone resorption. ${ }^{26}$

Dental age: It has been observed that, orthodontically treated teeth loose around $0.5 \mathrm{~mm}$ of its length. Those teeth with incomplete root formation undergoes less root desorption compared to those which with completely formed teeth. The incompletely formed root acquires further development due to orthodontic treatment and reaches their normal root length. ${ }^{26}$

Gender: Studies have shown that gender do not have role in root resorption. Thus male and female have equal chance for the root resorption. ${ }^{26}$

Ethnicity: Epidemiological studies have shown that, root resorption is less common in Asian compared to White, Caucasian or Hispanics. ${ }^{27,29}$

Nutrition: Animal studies have shown that, lack of Calcium and vitamin $D$ in food increases the risk of root resorption. ${ }^{26}$ There is lack of human studies to support these findings.

Root resorption prior to orthodontic treatment: Orthodontists need to pay attention to the patients undergoing the treatment who have prior history of root resorption because patients with root resorption prior to orthodontic treatment are at higher risk for root resorption. ${ }^{26}$

Personal Habits: Habits such as bruxism, nail biting, tongue thrust associated with open bite as well as increased tongue pressure increases the risk of root resorption. ${ }^{26}$

Anomalies of position and number of teeth: Risk of root resorption increases in hypodontia and impacted teeth. ${ }^{30}$ since third molars are the most commonly impacted 
teeth, it might lead to the resorption of second molar root. ${ }^{31}$ Subsequently, maxillary canines are second most commonly impacted teeth; they can eventually induce root resorption of incisors and first premolars. ${ }^{32}$

Dental trauma: Trauma can lead to root resorption even without orthodontic treatment. A published literature has shown that completely assimilated transplanted tooth exhibits the orthodontic force like normal tooth. ${ }^{30}$

Endodontically treated teeth: Some literatures illustrate that endodontically treated teeth exhibit resistance to root resorption. Thus hypothesis has been proposed that endodontically treated teeth are less susceptible to root resorption; however during the treatment root resorption often occurs. The quality of endodontic treatment is indespensible. ${ }^{23,26}$

Alveolar bone density: Some studies state that, during orthodontics treatment increased density of alveolar bone increases the risk of root resorption. However other studies emphasized the role of bone density in tooth movement but showed no relation in root resorption. ${ }^{23}$

\section{Mechanical factors}

Orthodontic appliances: Among fixed and removable appliances, fixed appliances has been reported to induce higher risk for root resorption. ${ }^{26}$ In the treatment of Angle Class II malocclusion; root resorption was diagnosed less in children undergoing two phase treatment (functional removable appliance and fixed appliance) compared to fixed orthodontic appliance alone. ${ }^{33}$ Long use of aesthetic bracket induce root resorption than the metallic brackets. ${ }^{34}$ Use of Class II elastics has shown higher risk of root resorption of mandibular molar distal roots. ${ }^{26}$

Tooth extraction: Role of tooth extraction in root resorption is often controversial though root resorption rates are higher in patients who have undergone several teeth extractions than those with no extraction..$^{35}$

Type of orthodontic tooth movement: Tooth movement during orthodontic intrusion induce root resorption. After the application of the same magnitude of continuous intrusion forces, it executes root resorption four times greater than that of the continuous extrusions. Tooth rotation however, causes only minor injuries of the periodontal tissues, which is most common in single-rooted teeth. ${ }^{26}$

Orthodontic force: Orthodontic force causes microtrauma of the periodontal ligaments leading to activation of the inflammatory cells. Heavy forces results more root resorption than the lighter forces. ${ }^{27}$ Continuous force leads to root resorption more commonly than the intermittent force. Intermittent force allows reorganization of periodontal ligaments and helps in restoration of blood circulation however the continuous force leaves no time. ${ }^{36}$

\section{Diagnosis of apical root resorption}

The most commonly used tool to diagnose root resorption is conventional radiography using panaromic and periapical views. ${ }^{26}$ It is recommended that radiographic investigation need to be carried out in first three to six months and every year after placement of the appliances. $\mathrm{CBCT}$ is the most sensitive imaging modality for diagnostic and prognostic assessment of apical resorption; hence it is the most promising tool. ${ }^{37,38}$ Further studies is warranted to outline the safety and cost effectiveness of $\mathrm{CBCT}$ in orthodontic patients.

\section{Long-term outcome of apical root resorption}

Root resorption affect maxillary incisors more frequently and to a more severe extent compared to rest of the dentition. ${ }^{39}$ According to the study, increased mobility occurs only in the teeth with extremely resorbed roots with very short root length. The mobility was minimal and non-significant in teeth with longer root lengths. The length of the follow-up period did not influence severity of root resorption, which implies that root resorption process does not progress following the termination of treatment. ${ }^{40}$

\section{Prevention and management strategies}

The clinicians being sensitive to root resorption; are required to counsel patients and their parents about the possible consequences of orthodontic treatment. Before initiating the treatment, clinicians need to take history with thorough investigation of potential predisposing factors and clinical conditions. Despite unclear understanding of the genetic susceptibility, it is recommended that patients with the positive parental history need to be investigated carefully correlating with the clinical data. As the heavy forces and intrusive tooth movement aggravate the resorption; it is recommended to apply the light force. In case of severe root resorption; it is essential to reassess the patient and minimize the underlying cause. The fixed appliances need to be designed carefully as per the appropriateness of the patients to prevent further resorption. The maintenance of the oral hygiene is very important and the patient needs to be well informed to maintain good oral hygiene to prevent periodontitis. For patients having extreme resorption; appropriate endodontic treatment is recommended. ${ }^{41}$ 
The literature has shown that the orthodontic patients are likely to suffer root resorption. It is important to understand the role of orthodontics in resulting root resorption. Despite the advances on genetic basis of bone resorption; advancement in clinical care regarding the bone resorption is yet to be unveiled. There is need of a large scale multi-centred clinical studies to understand biology, detection, and treatment of this undesired complication.

\section{REFERENCES}

1. Watson I. Tooth Integrity in orthodontics. Transcript of New Zealand Association of Orthodontists Annual Conference 2004.

2. Ngan, DC, Kharbanda OP, Byloff FK, Darendeliler MA. The genetic contribution to orthodontic root resorption: A retrospective twin study. Aus Orthod J. 2004; 20:1-9.

3. Bakland LK. Root Resorption. Dent Clin North Am. 1992; 36:491-507.

4. Fuss Z, Tsesis I, Lin S. Root resorption: Diagnosis, classification and treatment choices based on stimulation factors. Dent Traumatol. 2003; 19:175-82.

5. Robert-Harry D, Sandy J. Orthodontic tooth movement. Brit Dent J. 2004; 196:391-4.

6. Artun J, Smale I, Behbehani F, Doppel D, Van't Hof M, Kuijpers-Jagtman AM. Apical root resorption six and twelve months after initiation of fixed orthodontic appliance therapy. Angle Orthod. 2005; 75(6):919-26.

7. Rabinowitch BZ. Internal resorption. Oral Surg Oral Med Oral Pathol. 1957; 10(2):193-206.

8. Walton RE, Leonard LA. Cracked tooth: An etiology for "idiopathic" internal resorption? J Endod. 1986; 12(4):167-9.

9. Rabinowitch BZ. Internal resorption. Oral Surg Oral Med Oral Pathol. 1957; 10(2):193-206.

10. Benenati FW. Root resorption: Types and treatment. Gen Dent. 1997; 45(1):42-5.

11. Ne RF, Witherspoon DE, Gutmann JL. Tooth resorption. Quintessence Int. 1999; 30(1):9-25.

12. Wedenberg C, Zetterqvist L. Internal resorption in human teeth: A histological, scanning electron microscopic and enzyme histochemical study. J Endod. 1987; 13(6):255-9.

13. Henry JL, Weinmann JP. The pattern of resorption and repair of human cementum. J Am Dent Assoc. 1951; 42(3):270-290.

14. Massler M, Malone AJ. Root resorption in human permanent teeth: a roentographic study. Am J Orthod. 1954; 40(8):619-33.

15. Trope M. Clinical management of the avulsed tooth. Dent Clin North Am. 1995; 39(1):93-111.

16. Heithersay GS. Management of tooth resorption. Aus Dent J. 2007; 52(Suppl-1):105-21.

17. Andreasen FM. Transient apical breakdown and its relation to color and sensibility changes after luxation injuries to teeth. Endod Dent Traumatol. 1986; 2(1):9-19.

18. Brezniak N, Wasserstein A. Root resorption after orthodontic treatment: Part 2. Literature review. Am J Orthod Dentofac Orthop. 1993; 103(2):138-46.

19. Topkara A, Karaman I A, Kau CH. Apical root resorption caused by orthodontic forces: A brief review and a long-term observation. Euro J Dent. 2012; 6:445-53.

20. Sameshima GT, Sinclair PM. Predicting and preventing root resorption: Part I. Diagnostic factors. Am J Orthod Dentofacial Orthop. 2001; $119(5): 505-10$.

21. Artun J, Smale I, Behbehani F, Doppel D, Van't Hof M, Kuijpers-Jagtman AM. Apical root resorption six and 12 months after initiation of fixed orthodontic appliance therapy. Angle Orthod. 2005; 75(6):919-26.

22. M. Motokawa, T. Sasamoto, M. Kaku. Association between root resorption incident to orthodontic treatment andtreatment factors. Euro J Orthod. 2012; 34(3):350-6.

23. Hartsfield JK Jr, Everett ET, Al-Qawasmi RA. Genetic factors in external apical root resorption and orthodontic treatment. Crit Rev Oral Biol Med. 2004; 15(2):1 15-22.

24. Taithongchai R, Sookkorn K, Killiany DM. Facial and dentoalveolar structure and prediction of apical root shortening. Am J Orthod Dentofac Orthop. 1996; 110(3):296-302.

25. Mahida K, Agrawal C, Baswaraj H, Tandur AP, Patel B, Chokshi H. Root resorption: An abnormal consequence of orthodontic treatment. Int J Contemp Dent. 2015; 7-9.

26. Brezniak N. Root resorption after orthodontic treatment. Part II. Literature review. Am J Orthod Dentofac Orthop.1993; $103: 138-46$.

27. Hartsfield J. Genetic factors in external apical root resorption and orthodontic treatment. Crit Rev Oral Biol Med. 2004 ; $15: 115-22$.

28. Killiany DM. Root resorption caused by orthodontic treatment: Review of literature from 1998-2001 for evidence. Prog Orthod.2002; 3:2-5.

29. Sameshima GT, Sinclair PM. Prediction and prevention root resorption: Part I. Diagnostic factors. Am J Orthod Dentofacial Orthop.2001; 119:505-10.

30. Brezniak N. Orthodontically induced inflammatory root resorption. Part II: The clinical aspects. Angle Orthod. 2002;72:180-4

31. Tabiat-Pour S. Root resorption of a maxillary permanent first molar by an impacted second premolar. Br Dent J. 2007; $202: 261-2$.

32. Kokich VG RR Sr. Restoration and retention or maxillary anteriors with severe root resorption. J Am Dent Assoc. 2002; 133:67-71.

33. Brin I. External apical root resorption in Class II malocclusion: A retrospective review of 1-versus 2-phase treatment. Am J Orthod Dentofac Orthop. 2003; 124:151-6. 
34. Nigul K, Jagomagi T. Factors related to apical root resorption of maxillary incisors in orthodontic patients. Stomatologija Baltic Dent Maxillofac J. 2006; 8:76-9.

35. Travess H. Orthodontics. Part 6: Risks in orthodontic treatment. Br Dent J. 2004; 196:71-7.

36. Maltha JC, van Leeuwen EJ, Dijkman GE, Kuijpers-Jagtman AM. Incidence and severity of root resorption inorthodontically moved premolars in dogs. Orthod Craniofac Res. 2004; 7:115-21.

37. Dudic A, Giannopoulou C, Leuzinger M, Kiliaridis S. Detection of apical rootresorption after orthodontic treatment by using panoramic radiography and cone-beam computed tomography of super-high resolution. Am J Orthod Dentofac Orthop. 2009; 135:434-7.

38. Alqerban A, Jacobs R, Fieuws S, Willems $G$. Comparison of two cone beam computed tomographic systems versus panoramic imaging for localization of impacted maxillary canines and detection of root resorption. Eur J Orthod. 201 1; 33:93-102.

39. Remington DN, Joondeph DR, Artun J, Riedel RA, Chapko MK. Long-term evaluation of root resorption occuring during orthodontic treatment. Am J Orthod Dentofac Orthop. 1989; 96:43-6.

40. Jonsson A, Malmgren $\mathrm{O}$, Levander E. Long-term follow-up of tooth mobility in maxillary incisors with orthodontically induced apical root resorption. Eur J Orthod. 2007; 29:482-7.

41. Topkara A. External apical root resorption caused by orthodontic treatment: A review of the literature. Euro J Paed Dent. 2011;12(3):1636. 